\title{
BMJ Open Modelling-based evaluation of the costs, benefits and cost-effectiveness of multipathogen point-of-care tests for sexually transmitted infections in symptomatic genitourinary medicine clinic attendees
}

\author{
Susie E Huntington, ${ }^{1}$ Richéal M Burns, ${ }^{1,2}$ Emma Harding-Esch, ${ }^{3,4}$ \\ Michael J Harvey, ${ }^{1}$ Rachel Hill-Tout, ${ }^{5}$ Sebastian S Fuller, ${ }^{4}$ Elisabeth J Adams, ${ }^{1}$ \\ S Tariq Sadiq $3,4,5$
}

To cite: Huntington SE, Burns RM, Harding-Esch E, et al. Modelling-based evaluation of the costs, benefits and cost-effectiveness of multipathogen point-of-care tests for sexually transmitted infections in symptomatic genitourinary medicine clinic attendees. BMJ Open 2018;8:e20394. doi:10.1136/ bmjopen-2017-020394

- Prepublication history and additional material for this paper are available online. To view these files, please visit the journal online (http://dx.doi. org/10.1136/bmjopen-2017020394).

EJA and STS are joint senior authors.

Received 31 October 2017 Revised 16 April 2018 Accepted 21 May 2018

Check for updates

(c) Author(s) (or their employer(s)) 2018. Re-use permitted under CC BY-NC. No commercial re-use. See rights and permissions. Published by BMJ.

For numbered affiliations see end of article.

Correspondence to

Dr S Tariq Sadiq;

ssadiq@sgul.ac.uk

\section{ABSTRACT}

Objectives To quantify the costs, benefits and costeffectiveness of three multipathogen point-of-care (POC) testing strategies for detecting common sexually transmitted infections (STIs) compared with standard laboratory testing.

Design Modelling study.

Setting Genitourinary medicine (GUM) services in England.

Population A hypothetical cohort of 965988 people, representing the annual number attending GUM services symptomatic of lower genitourinary tract infection. Interventions The decision tree model considered costs and reimbursement to GUM services associated with diagnosing and managing STIs. Three strategies using hypothetical point-of-care tests (POCTs) were compared with standard care (SC) using laboratory-based testing. The strategies were: A) dual POCT for Chlamydia trachomatis (CT) and Neisseria gonorrhoeae (NG); B) triplex POCT for CT-NG and Mycoplasma genitalium (MG); C) quadruplex POCT for CT-NG-MG and Trichomonas vaginalis (TV). Data came from published literature and unpublished estimates.

Primary and secondary outcome measures Primary outcomes were total costs and benefits (quality-adjusted life years (QALYs)) for each strategy (2016 GB, £) and associated incremental cost-effectiveness ratios (ICERs) between each of the POC strategies and SC. Secondary outcomes were inappropriate treatment of STIs, onward STI transmission, pelvic inflammatory disease in women, time to cure and total attendances.

Results In the base-case analysis, POC strategy C, a quadruplex POCT, was the most cost-effective relative to the other strategies, with an ICER of $£ 36585$ per QALY gained compared with SC when using microcosting, and cost-savings of £26 451382 when using tariff costing. POC strategy $C$ also generated the most benefits, with 240467 fewer clinic attendances, 808 fewer onward STI transmissions and 235135 averted inappropriate treatments compared with SC.
Strengths and limitations of this study

- The main strength of this study is that it presents the first estimates of the potential public health impact of multipathogen point-of-care test strategies made possible by emerging diagnostic technologies.

- The model used inputs from multiple sources including published studies, published data (for costs), national surveillance data plus expert opinion and incorporated uncertainty in multiple input parameters by using a second-order Monte Carlo probabilistic sensitivity analysis and numerous scenarios were assessed in sensitivity analysis.

- The main limitations were the paucity of published data on treatment pathways including efficacy of treatment and gaps in treatment guidelines, which made building a representative standard care pathway problematic. There were few published data for some input parameters, for example, the percentage of patients who are presumptively treated without a microbiological result, or the percentage returning to clinic after initial treatment.

Conclusions Many benefits can be achieved by using multipathogen POCTs to improve STI diagnosis and management. Further evidence is needed on the underlying prevalence of STIs and SC delivery in the UK to reduce uncertainty in economic analyses.

\section{INTRODUCTION}

Continued high transmission rates of sexually transmitted infections (STIs) that cause treatable lower genital tract discharge syndromes (GDS) are a major public health concern. This causes significant reproductive-health long-term sequelae ${ }^{1-4}$ and frequently necessitates empirical therapy to minimise treatment 
delay, which often results in misdirected treatment, poor antimicrobial stewardship and the spread of antimicrobial resistance. ${ }^{56}$ Among the common causes of GDS, there were 107252 and 39696 diagnoses of Chlamydia trachomatis (CT) and Neisseria gonorrhoeae (NG), respectively, made in English genitourinary medicine (GUM) services in $2015,{ }^{7}$ with smaller numbers of Trichomonas vaginalis (TV) and Mycoplasma genitalium (MG) diagnoses.

The decision to give empirical antimicrobial therapy in symptomatic patients is usually guided by results of immediate microscopy of genital discharge, but this has low sensitivity, missing up to half of NG/TV infections in women ${ }^{8}$ and particularly poor specificity for predicting CT or MG. Accurate routine diagnosis, which requires laboratory-based nucleic acid amplification tests (NAATs), can take up to 2 weeks for results to be processed and most GUM services do not routinely conduct NAATs for MG or TV. Many patients are presumptively treated with either azithromycin or doxycycline, which are effective against CT but respectively cure only two-thirds and one-third of MG infections ${ }^{9}$ and neither antimicrobial is effective against TV. ${ }^{9}$

Emerging technologies are being developed that allow rapid and accurate point-of-care tests (POCTs) for multiple STIs, solutions which could address these challenges and help improve patient and public health outcomes. However, health services are under increasing financial pressure, and implementing new technologies may be prohibitively costly for both providers and commissioners of healthcare. There is currently only one commercially available rapid NAAT for CT and NG, which has equivalent performance to laboratory NAATs (Cepheid GeneXpert). ${ }^{10}$ Previous economic evaluations of NAAT POCTs for CT and NG indicate that they are likely to provide a cost-effective strategy for screening GUM attendees. ${ }^{511}$ To inform decision making by groups developing multipathogen POCTs and clinics which would likely use such tests, we assessed costs, benefits and cost-effectiveness of three testing strategies using hypothetical NAAT POCTs for A) CT-NG, B) CT-NG-MG and C) CT-NG-MG-TV compared with laboratory-based CT-NG NAAT testing.

\section{METHODS}

\section{Creating a model structure}

A decision tree model was constructed using Microsoft Excel (V.2016) to simulate a hypothetical cohort of people with symptoms of a lower genitourinary tract infection attending English GUM services. The basecase or primary analysis, using assumptions provided in online supplementary table 1 , compared complete pathway costs of three point-of-care (POC) strategies with the current practice of using microscopy plus a laboratory CT-NG NAAT. The POC strategies used microscopy plus a hypothetical POCT that provides results in $30 \mathrm{~min}$ for A) CT-NG; B) CT-NG-MG; C) CT-NG-MG-TV. Microscopy was used in all POC strategies as it can detect other conditions and infections not covered by the POCTs, as well as diagnosing NG and TV. The strategies were chosen in response to recent developments in STI POCT technology, reflecting pathogen configurations and performance characteristics. ${ }^{12} 13$

\section{Outcomes}

Primary outcomes were total costs and benefits, measured by quality-adjusted life years (QALYs) for each strategy and associated incremental cost-effectiveness ratios (ICERs, Eq. (1)) between each of the POC strategies and standard care (SC). The ICER provides information on the additional cost per unit of additional benefit between an option and the next less expensive alternative.

$$
I C E R x=\frac{\text { Cost }_{P O C_{x}}-\text { Cost }_{\text {Current }} \text { Practice }}{Q A L Y_{P O C x}-Q A L Y_{\text {Current Practice }}}
$$

Secondary outcomes assessed the wider health benefits and included inappropriate treatment of STIs (defined as unnecessary treatment of people with no STI plus incorrect treatment for people with an STI), onward transmission of the four STIs, pelvic inflammatory disease (PID), a serious complication of CT, NG and MG infections in women, time to cure and total GUM attendances.

\section{Patient pathways}

Three senior clinicians at St George's University Hospitals NHS Foundation Trust, London outlined current and hypothetical POC pathways for symptomatic patients (figure 1). We assumed that treatment pathways did not vary by subgroup but that MSM had diagnostic tests from three anatomical sites (genital, pharyngeal and rectal) at initial visit and MSW and women only had genital swabs. The model accounted for single or dual infections with NG, CT, MG and/or TV.

The cost of treating PID was included in the model but other long-term complications associated with STI infection (eg, infertility) and adverse drug events associated with treatment were not considered. The proportion of patients lost-to-follow-up (LTFU) was estimated. It was assumed that anyone remaining in follow-up consented to diagnostic testing and, if diagnosed, accepted treatment. The decision framework assumed that individuals had a maximum of three follow-up visits and then exited the model. Drug resistance was not considered. The time horizon for the model was 56 days. It was assumed that treatment was started on the day of diagnosis.

\section{Epidemiology and clinical parameters}

A short online survey, distributed to members of the British Association for Sexual Health and HIV (BASHH), was used to collect data on the percentage of patients attending GUM services that are symptomatic, the percentage of patients returning after initial visits as well as MG testing protocols, as limited data were available from published literature. The survey was completed by 23 GUM clinicians, 10 from London and 13 from elsewhere in the UK (see online supplementary table 2 ). 


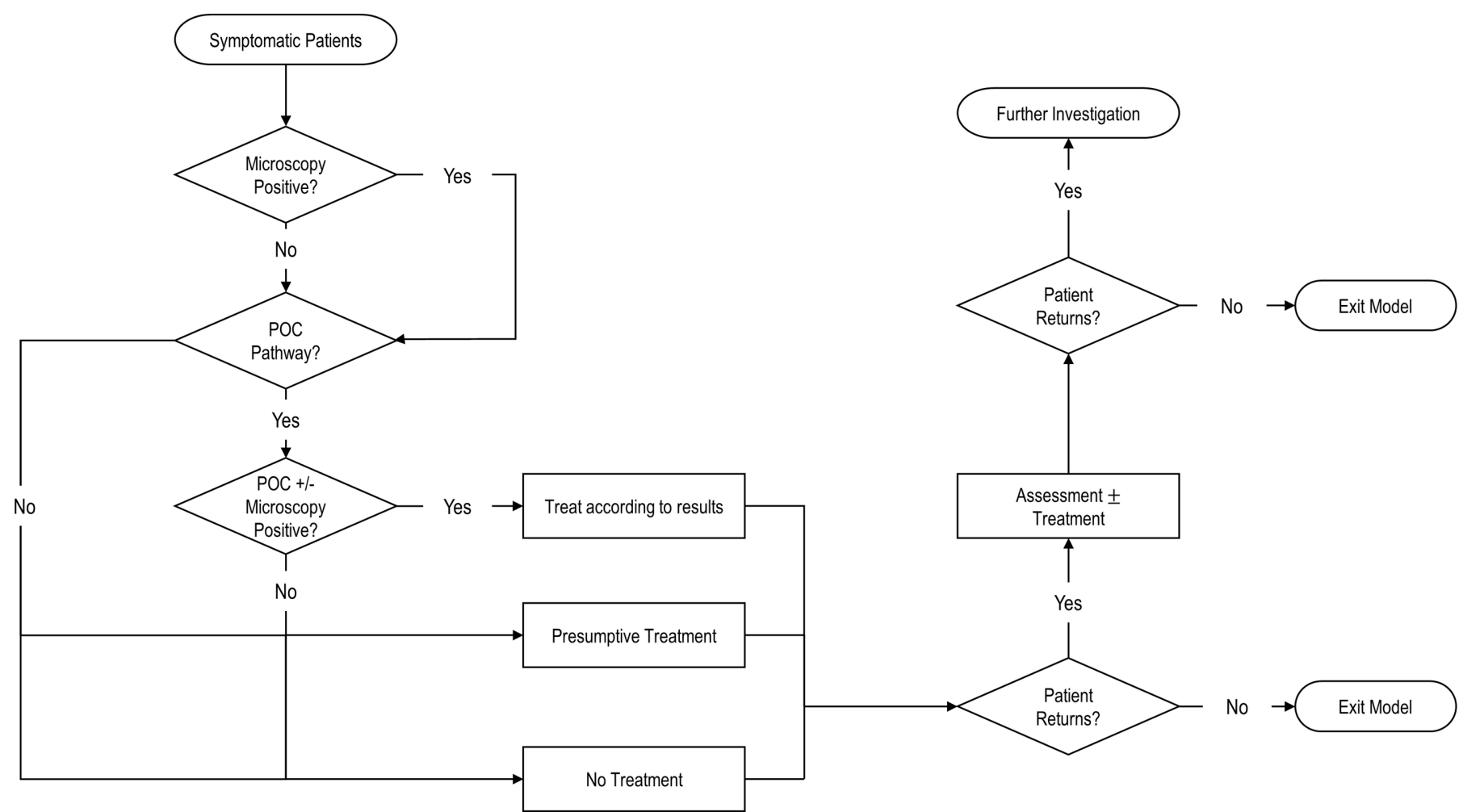

Figure 1 Simplified patient flow through the model. Standard care (SC): $50 \%$ of people not diagnosed with Neisseria gonorrhoeae (NG) or Trichomonas vaginalis (TV) by microscopy will be presumptively treated for Chlamydia trachomatis (CT). The treatment is effective against CT, $67 \%$ of Mycoplasma genitalium (MG) ${ }^{9}$ and against all other non-specific bacterial sexually transmitted infections (STIs) (ie, not CT/NG/MG/TV). We assume this treatment is not effective against TV or NG. Incorrectly treated patients with NG infection will be diagnosed by nucleic acid amplification test, and return (minus those lost-to-follow-up) to receive treatment. Other returning patients may receive presumptive treatment for MG and TV. Point-of-care (POC) strategy A: $50 \%$ of people not diagnosed by microscopy or point-of-care test (POCT) would be presumptively treated for MG and TV. We assume that this treatment is effective against CT, MG, TV and against all other non-specific bacterial STIs. We assume that this treatment is not effective against NG. Patients not initially presumptively treated but who return to the clinic are then presumptively treated with MG and TV treatment. POC strategy B: $50 \%$ of people not diagnosed by microscopy or POCT would be presumptively treated for TV. We assume this treatment is effective against TV, and against all other non-specific bacterial STIs. We assume this treatment is not effective against CT, MG or NG. POC strategy C: $100 \%$ of people who are not diagnosed by microscopy or POC would be presumptively treated using azithromycin. We assume this treatment is effective against CT, $67 \%$ of $\mathrm{MG}^{9}$ and against all other non-specific bacterial STIs. We assume this treatment is not effective against TV or NG. If any treated patients return, they will be categorised as 'investigate further'. The cost of the 'investigate further' is the cost of a standard return appointment that includes microscopy.

The total annual number of people attending English GUM services for STI testing was obtained from national surveillance data (GUMCAD 2015 data). ${ }^{7}$ The number of GUM attendees who were symptomatic was then calculated using the median percentages reported in the clinician survey (50\% of 1181574 for women; $40 \%$ of 647661 for men-who-have-sex-with-women (MSW) and $50 \%$ of 232274 for men-who-have-sex-with-men (MSM)). The model simulated a total of 965988 symptomatic attendees and by subgroup, 590787 women, 259064 MSW and 116137 MSM. We assumed that hypothetical POCTs had similar sensitivity and specificity to the best performing currently available CT-NG POCTs. ${ }^{10}$ The prevalence of CT, NG, MG and TV in GUM attendees (table 1) was estimated using published studies and preliminary findings from the PRECISE study, ${ }^{14}$ a study evaluating POCTs conducted by St George's, University of London and Public Health England.

\section{Patient and public involvement}

There was no involvement from patients or the public in the design of the evaluation.

\section{Cost and utility parameters}

All costs are given in 2015/2016 prices (GB, £) and inflated to $2015 / 2016$ costs when based on previous estimates using the Hospital and Community Health Services (HCHS) Inflation Indices 2015. ${ }^{15}$ The model considered two perspectives for costs: 1) costs to GUM services (microcosting) associated with testing and management of $\mathrm{NG} / \mathrm{CT} / \mathrm{MG} / \mathrm{TV}$ infections plus the cost of treating $\mathrm{PID}^{16}$ and 2) National Health Service (NHS) tariff reimbursements clinics would receive based on attendances (table 2). Microcosting was calculated by adapting an existing pathway model ${ }^{17}$ and includes the cost of staff time, diagnostic kit, drugs and other consumables. The tariff, an estimated average first and follow-up attendance 


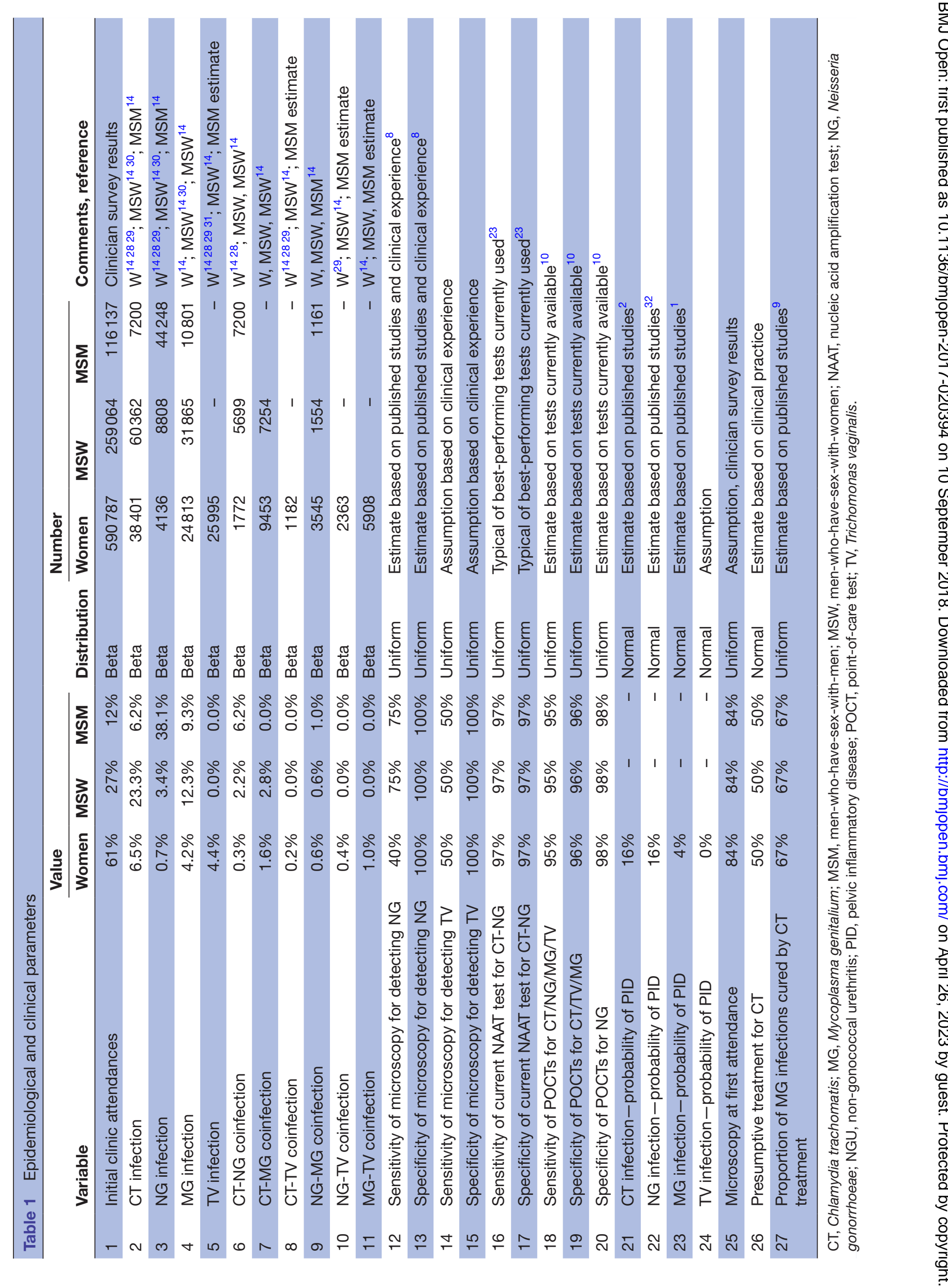


Table 2 Model input parameters: costs and utilities

Utilities

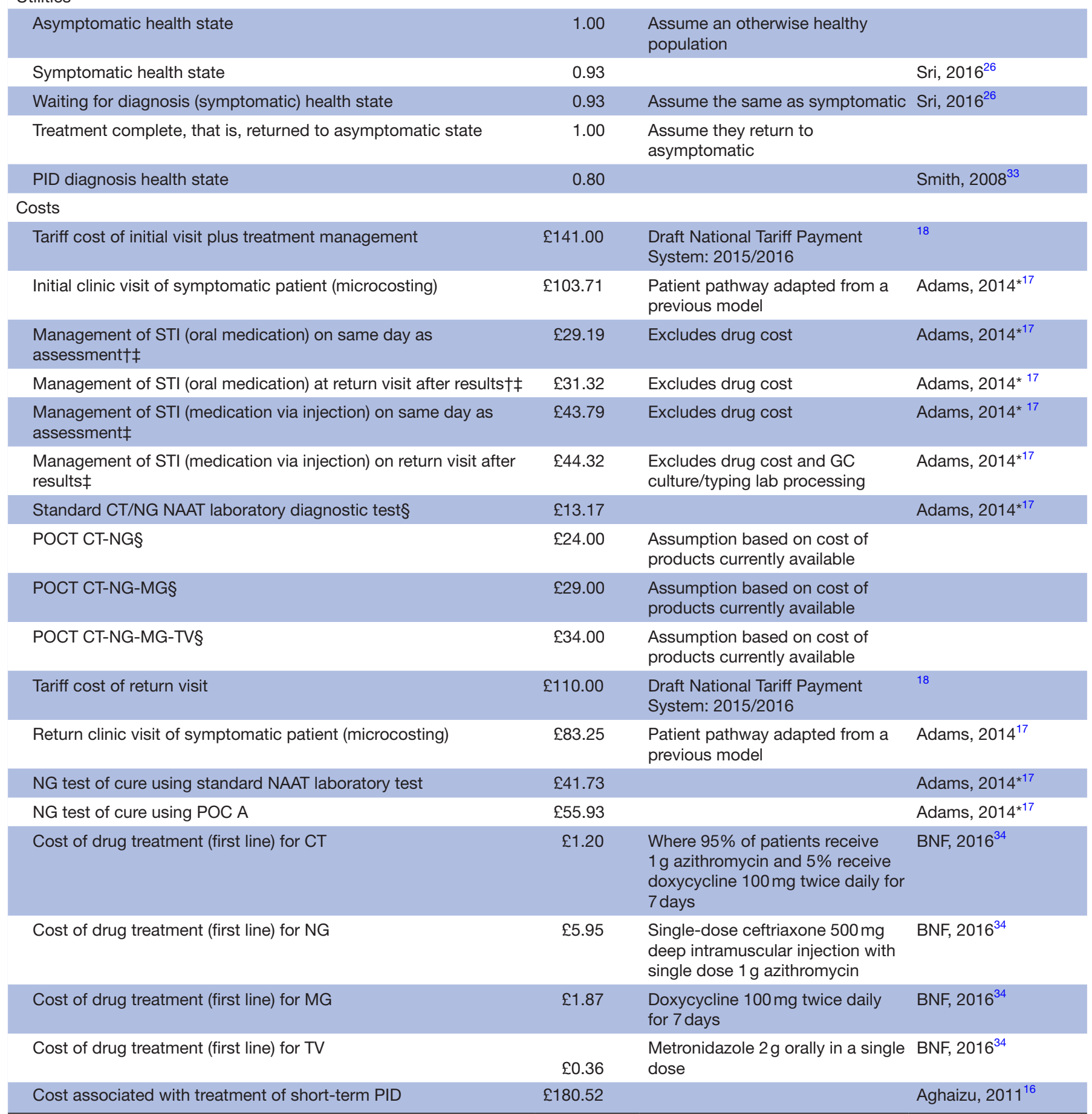

${ }^{*}$ Costs were inflated to $2015 / 2016$ costs using the Hospital and Community Health Services Inflation Indices 2015 produced by the Personal Social Services Research Unit. ${ }^{15}$ No data were available for inflation from 2014/2015 to 2015/2016 so it was assumed to be the same as between $2013 / 2014$ and 2014/2015. The UK consumer price index for health services shows similar annual growth in this sector from 2014 , which validates this assumption.

†The cost of management of MG/TV infection is assumed to be the same as the costs associated with management of CT infection. $\ddagger$ These costs vary due to the difference in administrative staff time for patient registration if the patient is treated on the same day or on a subsequent visit.

$\S M S M$ have samples from three sites (urethral, rectal, pharyngeal) tested at the initial visit, whereas women and MSW typically have one sample taken.

BNF, British National Formulary; CT, Chlamydia trachomatis; MG, Mycoplasma genitalium; NAAT, nucleic acid amplification test; NG, Neisseria gonorrhoeae; PID, pelvic inflammatory disease; POC, point-of-care; TV, Trichomonas vaginalis. 
cost used for commissioning and cross-charging, ${ }^{18}$ was estimated using the draft 2015/2016 NHS tariff for first and follow-up GUM attendances. Fixed tariffs are not affected by how treatment is administered (oral/intramuscular) or the number of tests performed.

Costs of implementing a change in practice, including training costs, were not considered. However, the unit costs used for staff time, which were considered, do incorporate the cost of training courses. ${ }^{15}$ Costs associated with testing and treating other causes of lower genitourinary tract symptoms were not considered.

Utilities, measures of health-related quality of life, were used in calculating QALYs for the health states (asymptomatic, symptomatic or awaiting test results) incorporated in the decision analytic framework (table 2).

\section{Scenario and sensitivity analysis}

As well as the base-case analysis, 32 further scenarios were assessed deterministically, that is, where one or more key parameters were varied based on changing the assumption of that parameter(s) while holding all others at the base-case level (see online supplementary table 3). Scenarios included: higher STI prevalence; lower sensitivity and specificity of POCTs; differing microscopy use; cheaper POCTs and different LTFU rates.

We performed a second-order Monte Carlo probabilistic sensitivity analysis (PSA), consistent with best practice, to estimate base-case ICERs. We converted model inputs from discrete values to distributions. For cost inputs, we used a normal distribution and varied each cost by $20 \%$ for the SD. We correlated costs of POCTs against the least expensive option to ensure test costs would change equivalently to the same degree with each simulation. For clinical parameters, we used beta and normal distributions for probabilities and uniform distributions for test performance. For utilities, normal distributions were used. The PSA included 5000 simulations and was performed using recommended procedures. ${ }^{19}$ Probabilities that strategies were cost-effective at a range of willingness-to-pay (WTP) thresholds per QALY gained were presented in cost-effectiveness acceptability curves (CEACs). CEACs were generated for each subgroup and the total population. Threshold costs for POCTs at which POC strategies would become cost-saving were calculated to the nearest $£ 0.25$.

\section{RESULTS}

Using microcosting, the estimated annual cost of testing and managing CT, NG, MG and TV infections in 965988 symptomatic individuals attending GUM was £113 058655 using SC. This was the cheapest strategy to clinical services, with POC strategies A, B and C being increasingly expensive at £118 704963 (5\% increase), £124 842003 (10.4\% increase) and 1125313136 (10.8\% increase), respectively (table 3). The opposite was true when using tariff costs, with SC being the most expensive for commissioners of sexual healthcare at $£ 172364138$, and POC strategy C being the least expensive, at $£ 145912757$ (table 3).

Most of the total care pathway cost was the initial attendance cost. In SC, total pathway costs for women were $£ 65122$ 097, of this 93\% ( $£ 60432050$ ) were initial

Table 3 The costs, QALYs, average time to cure, inappropriate treatment and follow-up visits in SC and three POC strategies for symptomatic people attending GUM services

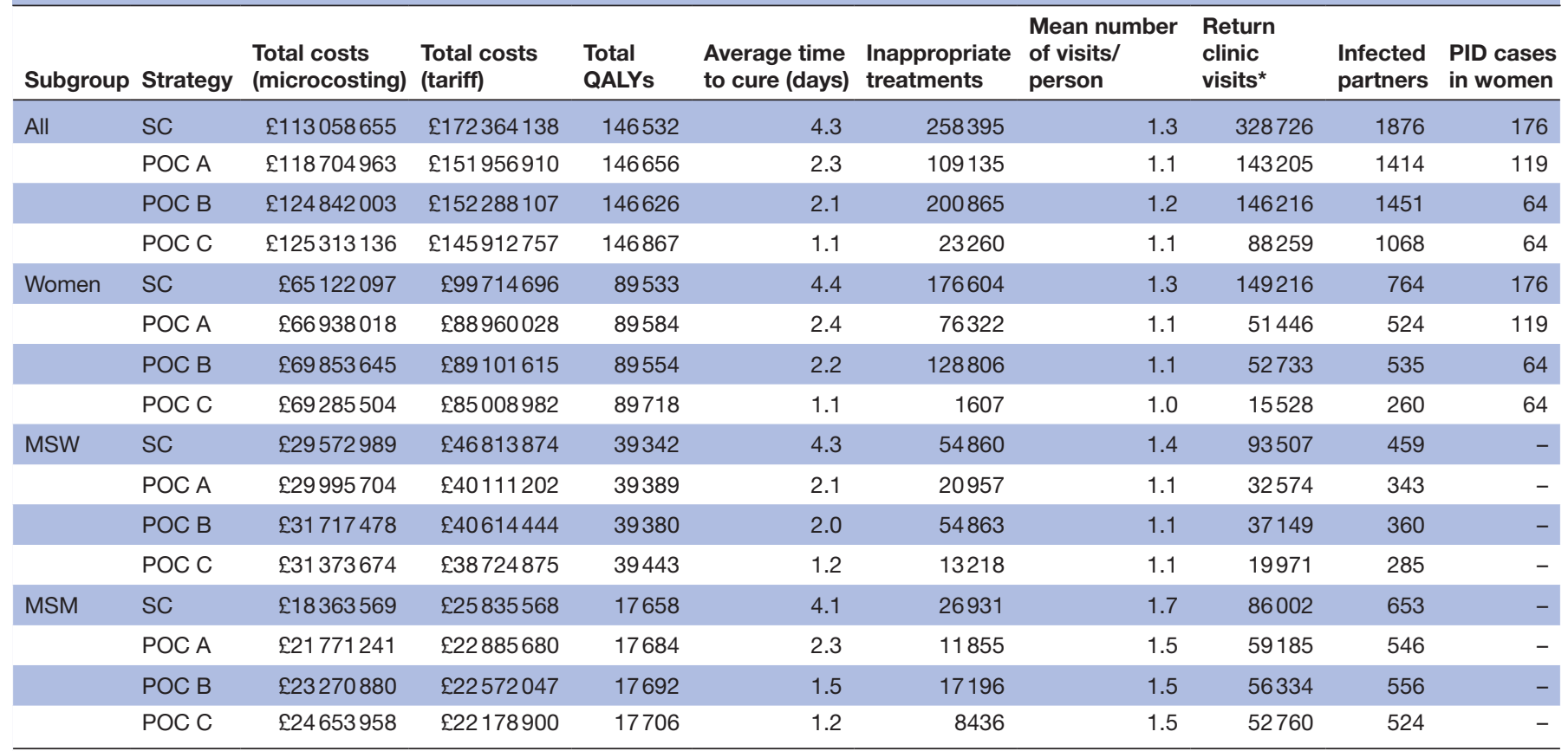

${ }^{*}$ Return clinic visit for results and treatment, a test of cure (routine for NG) or because they remain symptomatic.

GUM, genitourinary medicine; MSM, men-who-have-sex-with-men; MSW, men-who-have-sex-with-women; PID, pelvic inflammatory disease; POC, point-of-care; QALY, quality-adjusted life year; SC, standard care. 
Table 4 Cost differences for SC and POC strategies

\begin{tabular}{|c|c|c|c|c|c|c|}
\hline \multirow[b]{2}{*}{ Subgroup } & \multirow[b]{2}{*}{ Comparison } & \multirow[b]{2}{*}{ QALY difference } & \multicolumn{2}{|l|}{ Microcosting } & \multicolumn{2}{|l|}{ Tariff costs } \\
\hline & & & Cost difference & $\begin{array}{l}\text { ICER } \\
\text { (£/QALY gained) }\end{array}$ & Cost difference & $\begin{array}{l}\text { ICER } \\
\text { (£/QALY gained) }\end{array}$ \\
\hline \multirow{2}{*}{ All } & POC B vs SC & 94 & $£ 11783348$ & $£ 125197$ & $-£ 20076031$ & Cost-saving \\
\hline & POC C vs SC & 335 & $£ 12254482$ & $£ 36585$ & $-£ 26451382$ & Cost-saving \\
\hline \multirow[t]{4}{*}{ Women } & POC A vs SC & 51 & $£ 1815921$ & $£ 35608$ & $-£ 10754668$ & Cost-saving \\
\hline & POC B vs SC & 21 & $£ 4731548$ & $£ 222568$ & $-£ 10613081$ & Cost-saving \\
\hline & POC C vs SC & 185 & $£ 4163407$ & $£ 22448$ & $-£ 14705715$ & Cost-saving \\
\hline & POC B vs A & -30 & $£ 2915627$ & Dominated & $£ 141587$ & Dominated \\
\hline \multirow{3}{*}{ MSW } & POC C vs SC & 102 & $£ 1800685$ & $£ 17724$ & $-£ 8088999$ & Cost-saving \\
\hline & POC B vs A & -9 & $£ 1721773$ & Dominated & $£ 503242$ & Dominated \\
\hline & POC C vs B & 63 & $-£ 343804$ & Cost-saving & $-£ 1889570$ & Cost-saving \\
\hline \multirow[t]{5}{*}{ MSM } & POC A vs SC & 26 & $£ 3407672$ & $£ 130508$ & -£2 949888 & Cost-saving \\
\hline & POC B vs SC & 35 & $£ 4907312$ & $£ 141683$ & $-£ 3263521$ & Cost-saving \\
\hline & POC C vs SC & 48 & $£ 6290390$ & $£ 131319$ & $-£ 3656668$ & Cost-saving \\
\hline & POC B vs A & 9 & $£ 1499639$ & $£ 175909$ & $-£ 313633$ & Cost-saving \\
\hline & POC C vs B & 13 & $£ 1383078$ & $£ 104258$ & $-£ 393147$ & Cost-saving \\
\hline
\end{tabular}

ICER, incremental cost-effectiveness ratios; MSW, men-who-have-sex-with-women; MSM, men-who-have-sex-with-men; POC, point-ofcare; QALY, quality-adjusted life year; SC, standard care.

attendance costs. For POC strategy C, total pathway costs for women were $£ 69285504$, of which 98\% ( $£ 68041383$ ) were initial attendance costs.

POC strategy $\mathrm{C}$ provided most benefits to the full cohort. POC strategies A, B and C increased QALYs by 124, 94 and 335, respectively compared with SC (table 3 and table 4). Time to cure was shorter and the number of total clinic visits, onward STI transmissions and PID cases were fewer in POC strategies than in SC. Compared with SC, POC strategy C resulted in 240467 fewer clinic visits, 808 fewer onward STI transmissions, 235135 averted inappropriate treatments and 112 fewer cases of PID.

ICERs associated with the base-case model are presented in table 4. POC $\mathrm{C}$ highlights cost-effectiveness relative to other strategies yielding an ICER of $£ 36585$ per QALY gained compared with SC when using microcosting estimates. When incorporating NHS tariff costing, this represents a potential cost-savings of $£ 26451382$ relative to current practice.

CEACs are presented in figure 2. Using microcosting, for a WTP of $£ 30$ 000/QALY, the upper threshold adopted by the National Institute for Health and Care Excellence, ${ }^{20}$ SC had a $50 \%$ probability of being cost-effective overall, a $17 \%$ probability for women, a $2 \%$ probability for MSW and a $99 \%$ probability for MSM, relative to the POC strategies.
For a WTP of $£ 25000$, POC C dominates, that is, is cheaper and yields more benefits than the other POC strategies for the total cohort, women and MSW. For MSM, SC dominates all strategies across the thresholds examined. For MSW, POC A had $65 \%$ probability of being cost-effective at a WTP threshold of $£ 10000$ per QALY gained.

Results of 32 scenario analyses are summarised in online supplementary tables 4-7. The proportion of patients given presumptive treatment (scenarios 21-24) impacted costs hugely. When no presumptive treatment was given (scenario 21), the cost per QALY was $£ 7339$ and $£ 9092$ more than SC for POC strategies B and C, respectively, while POC strategy A was dominated by SC. When all patients without a diagnosis were presumptively treated for CT, MG or TV, according to which infections had been ruled out (scenario 24), POC strategies A and C. were dominated by SC and POC strategy B cost £64 300/ QALY more than SC.

When the utility score for being symptomatic was $10 \%$ less than base-case (scenario 25), cost per QALY was $£ 15627$ more for POC strategy C than for SC. If microscopy was no longer used in POC strategies (scenario 13), cost per QALY was $£ 16204$ and $£ 29594$ more than SC for POC strategies B and C, respectively, while POC strategy A was dominated by SC. 

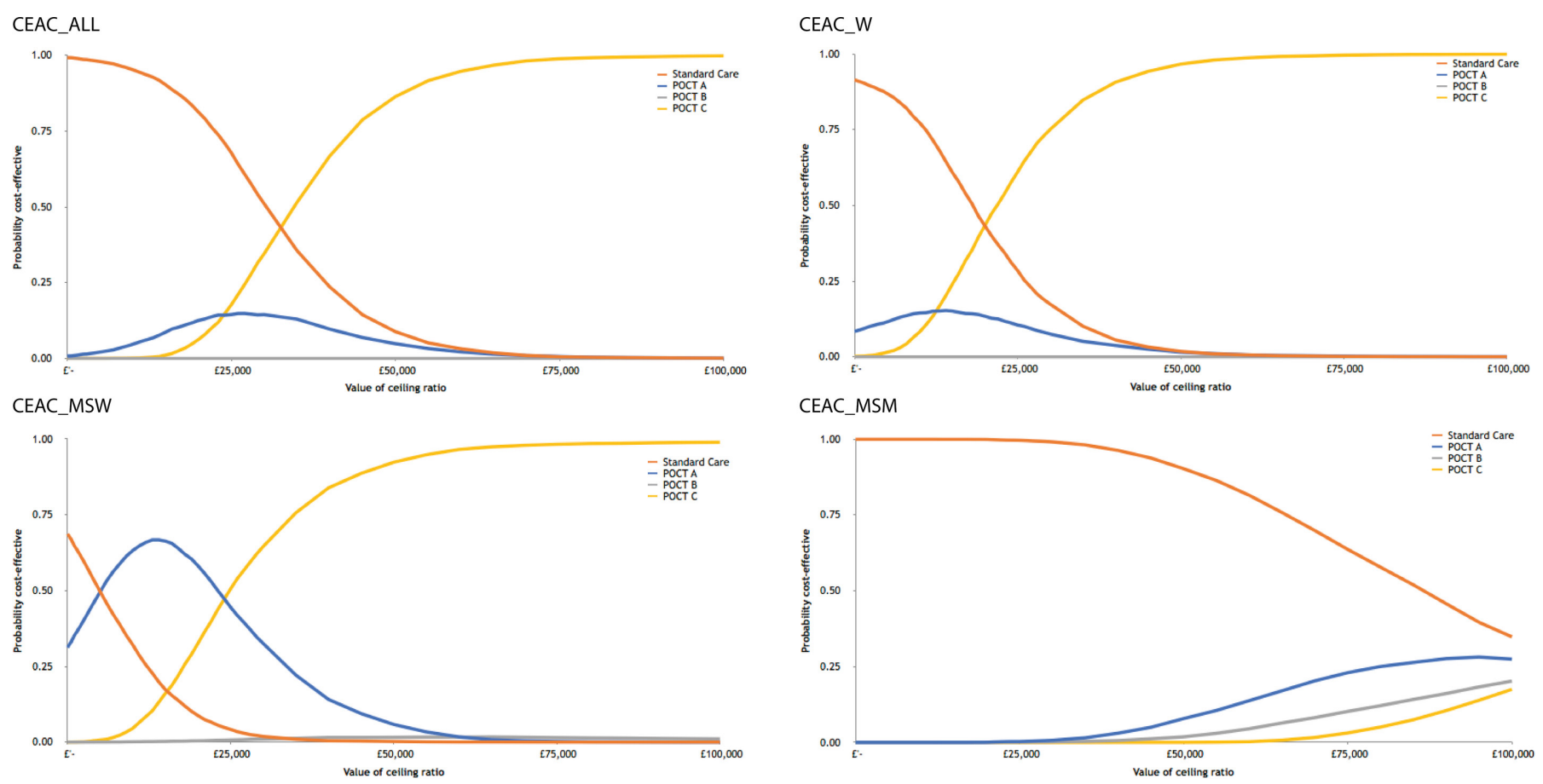

Figure 2 Cost-effectiveness acceptability curves (CEACs): point-of-care test (POCT) strategies vs standard care. MSM, menwho-have-sex-with-men; MSW, men-who-have-sex-with-women; W, women.

If $\mathrm{MG}$ and TV prevalence were as high as the estimated prevalence in the $\mathrm{USA}^{2122}$ (scenario 2), cost per QALY was $£ 20530$ and $£ 25548$ more than SC for POC strategies $\mathrm{B}$ and $\mathrm{C}$, respectively, while POC strategy $\mathrm{A}$ was dominated by SC. When POCTs were priced the same as the SC test (scenario 31), POC strategies B and C were costsaving (using microcosting).

Holding all other parameters constant using basecase assumptions, POC strategies would be cost-saving compared with SC, if POCTs cost £19.25, £13.50 and $£ 21.00$, respectively for POCTs A, B and C.

\section{DISCUSSION}

\section{Principle findings}

This study compared costs, benefits and cost-effectiveness of a laboratory-based CT-NG NAAT, with three POC strategies for: A) CT-NG; B) CT-NG-MG and C) CT-NGMG-TV. Using our initial assumptions for the total population using the microcosting approach to assess direct costs to healthcare services, each POC strategy cost more than SC, but yielded additional benefits. The proportion of patients given presumptive treatment and the cost of the POCT kit both impacted the costs hugely. Results indicated that different strategies would be cost-effective for different patient subgroups depending on the WTP threshold in place. POC strategy A was most cost-effective for MSW, POC strategy C for women and SC for MSM. Whether using different testing strategies on different patient groups would be practical or acceptable was beyond the scope of this research and requires further investigation. Different results were obtained when tariff reimbursement costs were used, with POC strategy C costing the least and providing the most benefits.

\section{Strengths and weaknesses}

Our analysis has several strengths. This is the first study to compare the cost-effectiveness of the three different STI POC strategies with SC. Comparable and consistent methods were used to construct a model with inputs from multiple sources including published studies, published data (for costs), national surveillance data plus expert opinion. The model incorporated uncertainty in multiple input parameters by using a second-order Monte Carlo PSA and numerous scenarios were assessed in sensitivity analysis.

Potential limitations should be considered. While it is thought that most UK clinicians follow the BASHH guidelines for STI testing and treatment, ${ }^{9}{ }^{23}$ there is diversity in the testing and management strategies employed to meet specific patient needs, both within and between clinics. This diversity, the paucity of published data on treatment pathways and gaps in treatment guidelines, made building a representative SC pathway problematic. There were few published data for some input parameters, for example, the percentage of patients who are presumptively treated without a microbiological result, the percentage returning to clinics after initial treatment and the percentage of patients attending with symptoms-all parameters which are likely to vary somewhat between clinics. We tried to overcome this using different scenario analysis and by collecting data using an online survey for GUM clinicians, although from a small sample size. Data from the survey showed high variation between 
clinics for some parameters. For example, the percentage of patients attending with symptoms ranged from $0 \%$ to $90 \%$. There were few published data on utilities and sexual behaviour while symptomatic with an STI, while waiting for test results and while being on treatment for an STI. Both qualitative and quantitative research is needed in this area to inform future health economic analyses and to understand preferences for and impact on patients of introducing POC testing.

The staffing and consumable cost data used in the model were average costs. In reality, different sites use staff of different grades and salaries to perform similar tasks and negotiate different costs for consumables, implying an inevitable variation in how cost-effective the different strategies would be for different sites.

The costs associated with long-term complications of STIs were not considered. ${ }^{24}$ This limitation means that the results are conservative, as the costs associated with SC are likely to be underestimated. However, the longterm benefits for a single round of testing were thought to be very low and to not have a major impact on the magnitude of results.

Comparing the results with other studies, POC pathways generated some cost-savings, primarily because patients had fewer return visits. However, these did not outweigh the higher cost of the POCTs compared with laboratory-based NAATs when estimating total pathway costs. This means that if POC testing was used, there would be expected overall cost increases based on the analysis using the microcosting approach. This differs from previous modelling, in which a CT-NG POCT was cost-saving compared with laboratory testing. ${ }^{5}$ In the model by Turner et al, the cost of a POC attendance was $£ 6.95$ cheaper than the cost of an SC attendance since the asymptomatic POC pathway was redesigned. In our model, the cost of attendance for a symptomatic patient was the same whether samples were sent to a laboratory or performed in clinic, since all symptomatic clinic attendees would require examination and microscopy.

\section{Implications for public health, clinicians and people using GUM services}

POC strategy C significantly decreased the numbers of inappropriate antibiotics given compared with SC, with over 235000 fewer inappropriate treatments. Many of these treatments are likely to include azithromycin, which when inappropriately given can encourage spread of macrolide antibiotic resistance in MG infections. ${ }^{6}$ POC strategy $\mathrm{C}$ also increased costs for health providers while decreasing tariff costs for commissioners, unless the 'freed-up' capacity enabled by POC strategy C was used for STI screening appointments and initial assessments for symptomatic people. Although this would likely have a positive impact on public health, given GUM services in England face increasing pressure on their services with $>2$ million new attendances annually, ${ }^{7}$ while local authorities equally face fiscal pressures, this work demonstrates the potential tensions between different cost-consequences for procurers and providers and public health benefits when considering implementation of novel health technologies. While there are no triplex or quadruplex POC STI tests currently on the market, there is at least one multiplex STI POC assay in development. ${ }^{25}$

For people using GUM services, there are numerous benefits to POC testing compared with SC. People would need fewer clinic visits, saving them time and reducing LTFU and reducing their costs such as out-of-pocket expenses and productivity losses. Receiving a diagnosis at the initial attendance reduces anxiety compared with waiting for test results. ${ }^{26}$ A negative result from a multipathogen POCT will support the development of clearer guidelines for clinicians, which could also reassure patients, particularly those with vague genital tract symptoms. Receiving effective treatment at initial attendance is likely to reduce the duration of symptoms and reproductive health sequelae. ${ }^{12}$

POCTs can also generate public health benefits from swifter diagnosis and treatment, for example, POC strategy $\mathrm{C}$ led to an estimated $43 \%$ fewer onward transmissions of STIs compared with SC. The ability to diagnose or rule out specific infections at first attendance enables accurate treatment and reduces inappropriate antibiotic use, crucial for good antibiotic stewardship, the economic benefits of which are substantial and well documented. ${ }^{27}$

\section{Unanswered questions and future research}

Although a rapid test for CT and NG is used in some GUM services, multipathogen configurations of CT-NGMG-TV POCTs are not currently available. As such, likely pathways for POC strategies had to be designed based on expert opinion. Published audits of GUM patient pathways would be a useful addition to the literature. To reduce uncertainty in economic analyses around STIs, further evidence is also needed on underlying STI prevalence and the risk of onward STI transmission.

Patient pathways and testing protocols vary between sites and different sites see different proportions of people from the three subgroups. As such, the costs of changing to a POC strategy will vary between sites as will the benefits and costs not considered in the model. Validated and easy to use computational tools for clinics and commissioners, which assess the economic impact of implementing any one of the POC strategies, using local population data, would increase understanding across stakeholders.

\section{CONCLUSION}

In conclusion, the results suggest that although potentially more expensive, a quadruplex test for CT-NG-MG-TV is more cost-effective than SC and the other testing strategies assessed, providing the most additional benefits to patients. Cost implications are driven by the cost of POCTs and would vary somewhat in different geographical areas due to differences in the subgroup mix and the prevalence of the four STIs. 
Author affiliations

${ }^{1}$ Aquarius Population Health, 58a Highgate High Street, London, UK ${ }^{2}$ Health Economics and Policy Analysis Centre (HEPAC), NUI Galway, Ireland ${ }^{3}$ HIV/STI Department, National Infection Service, Public Health England, 61 Colindale Avenue, London, UK

${ }^{4}$ St George's Institute for Infection and Immunity, Applied Diagnostic Research and Evaluation Unit, University of London, Cranmer Terrace, London, UK

${ }^{5}$ St George's University Hospitals NHS Foundation Trust, Blackshaw Road, Tooting, London, UK

Contributors All authors were involved in the conception and design of the research. STS, EH-E, RH-T and SSF provided input into current clinical practice relating to patient pathways in GUM. SEH estimated all parameter inputs and drafted the manuscript with support from EJA and MJH. RMB developed the model with input from EJA, SEH and MJH. All authors contributed to the interpretation of the model and approved the final version. STS acts as guarantor of the study.

Funding This work was supported by Innovate UK SBRI grant no. 971452, awarded to Atlas Genetics Ltd. Atlas Genetics paid the open access publication fee.

Disclaimer Atlas Genetics and Innovate UK had no role in the study design; in the collection, analysis and interpretation of data; in the writing of the report and in the decision to submit the article for publication.

Competing interests All authors have completed the Unified Competing Interest Form and declare financial support from Innovate UK; EJA, SEH, MJH are employees of Aquarius Population Health (APH), which reports grants from Innovate UK grant to Atlas Genetics, during the conduct of the study; other from Cepheid, St Georges University of London, Enigma Diagnostics and AstraZeneca, on STI and POC research outside the submitted work; RMB is a Lecturer and Programme Director of Economics at St. Angela's College Sligo/NUI Galway and an academic staff member of the Health Economics and Policy Analysis Centre (HEPAC) at NUI Galway, providing health economic support to Aquarius Population Health on an ad hoc consultancy basis. STS, EH-E, SSF are members of the Applied Diagnostic Research and Evaluation Unit at St George's, University of London, which has received funding from Atlas Genetics, Alere, Cepheid, SpeeDx and Sekisui. STS has received NIHR funding to develop a POCT with Atlas.

Patient consent Not required.

Provenance and peer review Not commissioned; externally peer reviewed.

Data sharing statement № additional data available. All data supporting the conclusions of the paper are included within the article or in the online supplementary material.

Open access This is an open access article distributed in accordance with the Creative Commons Attribution Non Commercial (CC BY-NC 4.0) license, which permits others to distribute, remix, adapt, build upon this work non-commercially, and license their derivative works on different terms, provided the original work is properly cited, appropriate credit is given, any changes made indicated, and the use is non-commercial. See: http://creativecommons.org/licenses/by-nc/4.0/.

\section{REFERENCES}

1. Oakeshott $P$, Aghaizu A, Hay $P$, et al. Is Mycoplasma genitalium in women the "New Chlamydia?" A community-based prospective cohort study. Clin Infect Dis 2010;51:1160-6.

2. Price MJ, Ades AE, De Angelis D, et al. Risk of pelvic inflammatory disease following Chlamydia trachomatis infection: analysis of prospective studies with a multistate model. Am J Epidemiol 2013;178:484-92.

3. Liu B, Roberts CL, Clarke M, et al. Chlamydia and gonorrhoea infections and the risk of adverse obstetric outcomes: a retrospective cohort study. Sex Transm Infect 2013;89:672-8.

4. Hitti J, Garcia P, Totten P, et al. Correlates of cervical Mycoplasma genitalium and risk of preterm birth among Peruvian women. Sex Transm Dis 2010;37:81-5.

5. Turner KM, Round J, Horner P, et al. An early evaluation of clinical and economic costs and benefits of implementing point of care NAAT tests for Chlamydia trachomatis and Neisseria gonorrhoea in genitourinary medicine clinics in England. Sex Transm Infect 2014:90:104-11.

6. Jensen JS, Bradshaw C. Management of Mycoplasma genitalium infections - can we hit a moving target? BMC Infect Dis 2015;15:343.

7. Public Health England. Sexually transmitted infections (STIs): annual data tables, 2006-2015. www.gov.uk/government/statistics/sexually- transmitted-infections-stis-annual-data-tables (accessed 20 Sep 2016).

8. Thorley N, Radcliffe K. The performance and clinical utility of cervical microscopy for the diagnosis of gonorrhoea in women in the era of the NAAT. Int J STD AIDS 2015;26:656-60.

9. Horner P, Blee K, O'Mahony C, et al. 2015 UK National guideline on the management of non-gonococcal urethritis. Int J STD AIDS 2016;27:85-96.

10. Herbst de Cortina S, Bristow CC, Joseph Davey D, et al. A systematic review of point of care testing for chlamydia trachomatis, Neisseria gonorrhoeae, and Trichomonas vaginalis. Infect Dis Obstet Gynecol 2016;2016:1-17.

11. Huang W, Gaydos CA, Barnes MR, et al. Comparative effectiveness of a rapid point-of-care test for detection of Chlamydia trachomatis among women in a clinical setting. Sex Transm Infect 2013;89:108-14.

12. Atlas Genetics. Atlas Genetics wins SBRI grant from Innovate UK. $2016 \mathrm{http}: / /$ atlasgenetics.com/130-atlas-genetics-wins-sbri-grantfrom-innovate-uk (accessed 6 Apr 2018).

13. Harding-Esch EM, Fuller SS, Chow C, et al. Performance of a prototype chlamydia and gonorrhoea recombinase polymerase amplification point-of-care test in three sexual health clinics: In. Eighth Meeting of the European Society for Chlamydia Research. Oxford, UK, 2016. www.escr2016.co.uk/programme.pdf. (accessed 4 Jan 2017).

14. S Tariq Sadiq personal communication. PRECISE preliminary results, 2016. http://preciseresearch.co.uk/

15. PSSRU. Unit Costs of Health and Social Care. 2015 www.pssru.ac. uk/project-pages/unit-costs/2015/ (accessed 29 Sep 2016).

16. Aghaizu A, Adams EJ, Turner K, et al. What is the cost of pelvic inflammatory disease and how much could be prevented by screening for chlamydia trachomatis? Cost analysis of the Prevention of Pelvic Infection (POPI) trial. Sex Transm Infect 2011;87:312-7.

17. Adams EJ, Ehrlich A, Turner KM, et al. Mapping patient pathways and estimating resource use for point of care versus standard testing and treatment of chlamydia and gonorrhoea in genitourinary medicine clinics in the UK. BMJ Open 2014;4:e005322.

18. Monitor. 2016/17 National Tariff Payment System: draft prices. 2016 www.gov.uk/government/publications/201617-national-tariffpayment-system-draft-prices (accessed 21 Jun 2016).

19. Briggs $\mathrm{AH}$, Weinstein MC, Fenwick EA, et al. Model parameter estimation and uncertainty analysis: a report of the ISPOR-SMDM Modeling Good Research Practices Task Force Working Group-6. Med Decis Making 2012;32:722-32.

20. National Institute for Health and Care Excellence. Judging whether public health interventions offer value for money. 2013 www.nice.org uk/advice/lgb10/chapter/judging-the-cost-effectiveness-of-publichealth-activities (accessed 31 Oct 2017).

21. Getman D, Jiang A, O'Donnell M, et al. Mycoplasma genitalium prevalence, coinfection, and macrolide antibiotic resistance frequency in a multicenter clinical study cohort in the United States. J Clin Microbiol 2016;54:2278-83.

22. Alcaide ML, Feaster DJ, Duan R, et al. The incidence of Trichomonas vaginalis infection in women attending nine sexually transmitted diseases clinics in the USA. Sex Transm Infect 2016;92:58-62.

23. Nwokolo NC, Dragovic B, Patel S, et al. 2015 UK national guideline for the management of infection with Chlamydia trachomatis. Int $J$ STD AIDS 2016;27:251-67.

24. Davies B, Turner KME, Frølund M, et al. Risk of reproductive complications following chlamydia testing: a population-based retrospective cohort study in Denmark. Lancet Infect Dis 2016;16:1057-64.

25. Khalil S. Innovate UK gives Atlas Genetics $£ 2 m$ contract for rapid diagnostic platform: Digital Health Online News Article, 2017. www. digitalhealth.net/2017/08/atlas-genetics-awarded-2m-innovate-ukcontract. (accessed 6 April 2018).

26. Sri T, Southgate E, Kerry SR, et al. Health-related quality of life and Chlamydia trachomatis infection in sexually experienced female inner-city students: a community-based cross-sectional study. Int J STD AIDS 2017;28:367-71.

27. Smith $\mathrm{R}$, Coast J. The true cost of antimicrobial resistance. BMJ 2013;346:f1493.

28. Shone J, Winter A, Jones BL, et al. A Scottish multi-centre service evaluation examining the prevalence and diagnosis of Trichomonas vaginalis in symptomatic women attending sexual health clinics. Int $J$ STD AIDS 2016;27.

29. Nathan B, Appiah J, Saunders P, et al. Microscopy outperformed in a comparison of five methods for detecting Trichomonas vaginalis in symptomatic women. Int J STD AIDS 2015;26:251-6.

30. Chalker VJ, Jordan K, Ali T, et al. Real-time PCR detection of the mg219 gene of unknown function of Mycoplasma genitalium in men 
with and without non-gonococcal urethritis and their female partners in England. J Med Microbiol 2009;58:895-9.

31. Lord E, Newnham T, Dorrell L, et al. Detecting asymptomatic Trichomonas vaginalis in females using the BD ProbeTec Trichomonas vaginalis $\mathrm{Q}^{\mathrm{x}}$ nucleic acid amplification test. Int J STD AIDS 2017;28:357-61.

32. Reekie J, Donovan B, Guy R, et al. Hospitalisations for pelvic inflammatory disease temporally related to a diagnosis of
Chlamydia or gonorrhoea: a retrospective cohort study. PLoS One 2014;9:e94361.

33. Smith KJ, Tsevat J, Ness RB, et al. Quality of life utilities for pelvic inflammatory disease health states. Sex Transm Dis 2008;35:307-11.

34. BNF September 2016. https://www.evidence.nhs.uk/formulary/bnf/ current (accessed 29 Sep 2016). 